\title{
GCU
}

Glasgow Caledonian

University

University for the Common Good

\section{A revised model for calculating HV cable sheath current under short-circuit fault condition and its application for fault location - Part 1: the revised model}

Li, Mingzhen; Zhou, Chengke; Wenjun, Zhou

Published in:

IEEE Transactions on Power Delivery

DOI:

10.1109/TPWRD.2019.2918159

Publication date:

2019

Document Version

Author accepted manuscript

Link to publication in ResearchOnline

Citation for published version (Harvard):

$\mathrm{Li}, \mathrm{M}$, Zhou, C \& Wenjun, Z 2019, 'A revised model for calculating HV cable sheath current under short-circuit fault condition and its application for fault location - Part 1: the revised model', IEEE Transactions on Power Delivery, vol. 34, no. 4, pp. 1674-1683. https://doi.org/10.1109/TPWRD.2019.2918159

\section{General rights}

Copyright and moral rights for the publications made accessible in the public portal are retained by the authors and/or other copyright owners and it is a condition of accessing publications that users recognise and abide by the legal requirements associated with these rights.

Take down policy

If you believe that this document breaches copyright please view our takedown policy at https://edshare.gcu.ac.uk/id/eprint/5179 for details of how to contact us. 


\title{
A Revised Model for Calculating HV Cable Sheath Current under Short-circuit Fault Condition and Its Application for Fault Location Part 1: The Revised Model
}

\author{
Mingzhen Li, Student Member, IEEE, Chengke Zhou, Senior Member, IEEE and Wenjun Zhou, \\ Senior Member, IEEE
}

\begin{abstract}
This two-part paper proposes a revised method for calculating HV (High Voltage) cable sheath currents, and for fault location of cross-bonded $\mathrm{HV}$ cable systems. The equivalent circuit and the cable impedance per unit length under shortcircuit fault conditions are revised from those under non-fault conditions. Part 1 of this paper proposes a revised phasor domain mathematical model for the sheath current calculation under fault conditions, following demonstration that short-circuit faults can cause changes in the equivalent circuit of the cable metal sheath, and causing changes in the cable impedance per unit length. The calculation results, as given in a case study, show that there exists a unique relation between the fault position and the sheath fault currents, making it possible to locate a fault by analyzing the characteristics of the sheath currents during a fault. Part 2 of the paper focuses on the fault location method via sheath current monitoring.
\end{abstract}

Index Terms-- Cable shielding, Circuit faults, Fault currents, Fault location, Transmission lines

\section{INTRODUCTION}

$\mathrm{W}$ ITH the continuous expansion of urban areas, the demand for electricity has been fast growing [1-3]. In order to meet the reliability and security requirements of urban power transmission, taking into account urban space and planning constraints, power cables have been widely adopted in urban power grids $[4,5]$. Compared with overhead lines, power cable has the advantages of high reliability, small footprint, and being less susceptible to the surrounding environment [6]. With the increase in adoption of cable for interconnection, the number of cable short-circuit faults has increased [7], adversely affecting the safe and stable operation of power grids, as an outage due to cable fault often takes longer to restore.

Currently two methods have primarily been adopted for fault location of $\mathrm{HV}$ cable systems. One is the impedancebased method, using fault voltage/current data collected from relay protection devices [8-10], and the other is the traveling wave method [11-14]. The theory of the impedance-based

M. Li and W. Zhou are with Wuhan University, Wuhan, 430072, China (e-mail: li-mingzhen@qq.com; wjzhou@whu.edu.cn).

C. Zhou is with Glasgow Caledonian University, Glasgow, G4 0BA, UK (e-mail: c.zhou@gcu.ac.uk). method is based on parameter identification, as the system parameters would change when a fault occurs [9]. Since the measured impedance may not be linearly proportional to fault distance [10], it is difficult to determine accurately the relationship of impedance versus distance. The theory of the traveling wave method is based on analysis of the propagation time of the transient wave associated with fault current [1114]. The problems associated with the traveling wave method lie with noise elimination and accurate identification of the wave head. The background of these two methods stems from the determination of location of an overhead line short-circuit fault.

Recently, there have been improvements to the impedancebased method and the traveling wave method. The improvements to impedance-based method mainly focused on the calculations for specific applications $[15,16]$, for example, the applications in double circuit lines [15], and in the identification for line faults [16]. Improvements to the impedance-based method are mainly in the algorithms [1719], e.g. making the fault signal easier to identify $[17,18]$ or finding other fault characteristics [19]. However, almost all these methods require detailed system topology including line and cable parameters which may not be readily available in practice.

The telegrapher's equations [20] have been widely used to describe the voltage and current of an electrical transmission line with distance and time under both fault condition and non-fault condition. The equations resulted from the transmission line model [20], which assumed that the conductors were composed of an infinite series of two-port elementary components, each representing an infinitesimally short segment of the transmission line. When power cables are considered, Wedepohl redefined the parameters in the telegrapher's equations [21], as shown in (1) and (2), where $\boldsymbol{V}$ and $\boldsymbol{I}$ are vectors of dimension $\mathrm{n}$ representing, respectively, the voltages and currents at a distance $\mathrm{x}$ along the cable system containing n metallic conductors. $\boldsymbol{Z}$ and $\boldsymbol{Y}$ are square matrices of dimension $\mathrm{n}$. Wedepohl presented in detail the matrix elements of $\boldsymbol{Z}$ and $\boldsymbol{Y}$ in [22], with each cable having two metallic conductors. Every matrix element is composed of admittances and impedances per unit length: these are determined by the cable configuration, the cable materials and 
the relative position between cables. However, short-circuit faults can cause changes in the equivalent circuit of the cable metal sheath and in the cable impedance per unit length.

$$
\begin{aligned}
& \frac{d \boldsymbol{V}}{d x}=-\boldsymbol{Z} \cdot \boldsymbol{I} \\
& \frac{d \boldsymbol{I}}{d x}=-\boldsymbol{Y} \cdot \boldsymbol{V}
\end{aligned}
$$

Part 1 of the paper presents the revised equivalent circuit of the cable metal sheath under fault conditions. It explains the changes in the cable impedance per unit length, and proposes a revised phasor domain mathematical model for calculating cable sheath currents of a cross-bonded cable system under fault conditions. Thereafter, it shows the variation of sheath currents with respect to the change in assumed fault location.

Part 2 of the paper proposes a fault location approach via sheath current monitoring. The approach includes two steps: first, the exact cable section where a fault occurs can be determined from changes in current values, as the sheath currents would flow in opposite direction at the two ends of the fault section, whilst they would flow in the same direction in a healthy section; second, the exact fault position in the fault section can be determined from the phase difference of the power frequency sheath currents at the two ends of the fault section.

\section{THE EQUivalent CiRCuIT OF CROSS-BONDED CABLE AND THE MODEL FOR SHEATH CURRENTS CALCULATION UNDER FAULT AND NON-FAULT CONDITIONS}

Cross-bonding is one of the main features in a $\mathrm{HV}$ cable system. To reduce the effects of unbalanced three-phase load on sheath currents, long HV cable circuits $(>1.2 \mathrm{~km})$ usually have their metal sheath or the conductors transposed every 400 500 meters, as shown in Fig. 1. It is to be noted that a whole cross-bonding section (referred to as "major section" in [23]) consists of three adjacent cable sections or cable segments (referred to as "minor sections" in [23]) of which the metal sheaths are cross-bonded. At both ends of a major section the metal sheath is directly connected to ground through grounding boxes G1 and G2. At joints J1 and J2 the sheaths are connected to ground via overvoltage limiters.

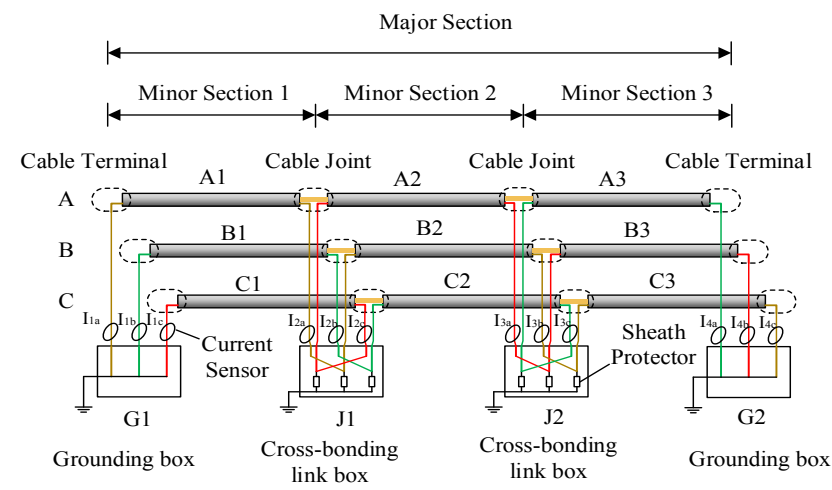

Fig. 1. The configuration of a cross-bonded HV cable system

The cross-section of a typical HV cable is shown in Fig.2, where $r_{1}$ is the core radius; $r_{2}$ is the main insulation outer radius; $r_{3}$ is the metal sheath outer radius, and $r_{4}$ is the jacket outer radius. Starting from the centre of the cable, the cable structure is composed of the core conductor, conductor shield, main insulation, insulation shield, metal sheath, and the jacket. As shown in Fig. 1, the two ends of the metal sheath of a major section are directly grounded. The equivalent circuit for sheath current calculation is shown in Fig. 3. The formula for determining sheath current is given in (3), where $I_{m 1}$ is the sheath current of the circuit made up of elements A1-B2-C3; $U_{a 1}$ is the induced electromotive force (emf) of the sheath section $\mathrm{A} 1 ; U_{b 2}$ is the induced emf of the sheath section B2; $U_{c 3}$ is the induced emf of the sheath section $\mathrm{C} 3 ; Z_{m a 1}$ is the equivalent impedance of sheath $\mathrm{A} 1 ; Z_{m b 2}$ is the equivalent impedance of sheath $\mathrm{B} 1$, and $Z_{m c 3}$ is the equivalent impedance of sheath C3. $R_{g 1}$ is the ground resistance at G1 and $R_{g 2}$ is the ground resistance at $\mathrm{G} 2$. It is to be noted that $U_{a 1}, U_{b 2}, U_{c 3}$ contain the induced emfs from other adjacent cable phases.

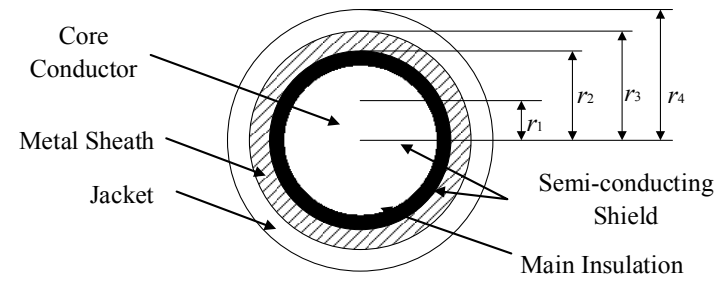

Fig. 2. The cross-section of a typical HV cable

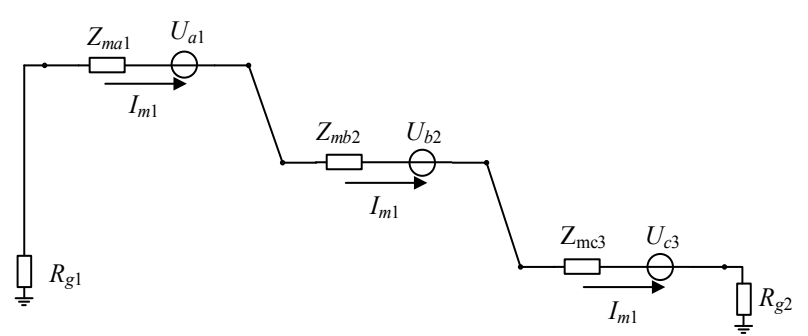

Fig. 3. The equivalent circuit for sheath currents calculation under non-fault conditions

$$
I_{m 1}=\frac{U_{a 1}+U_{b 2}+U_{c 3}}{Z_{m a 1}+Z_{m b 2}+Z_{m c 3}+R_{g 1}+R_{g 2}}
$$

The reason for proposing a new modelling method is to take into account that a fault causes changes in the equivalent circuit of the cable metal sheath, and in the cable impedance per unit length. During a fault, fault current flows from the core conductor through the metal sheath, and the inductance between the core conductor and the metal sheath of the fault loop should include both self-inductance and mutual inductance. For the example of when a short circuit fault occurs in section A1, the equivalent circuit of the fault loop is shown in Fig. 4, where $U_{a}$ is the equivalent voltage source of phase $\mathrm{A}, U_{A 1 S}$ is the equivalent induced emf on sheath section A1 (between the fault position and G1), $U_{A 1 R}$ is the equivalent induced emf on sheath section A1 (between the fault position and J1), $R_{f}$ is the fault resistance, $I_{f}$ is the fault current starting from the power source to the fault position. $I_{S}$ is the fault current starting from the fault position to G1; $I_{R}$ is the fault current starting from the fault position to G2. The equations of $I_{S}$ and $I_{R}$ are shown in (4) and (5), where $Z_{A 1 S}$ is the equivalent impedance of sheath section A1 (between the fault position and G1), and $Z_{A 1 R}$ is the equivalent impedance of sheath section $\mathrm{A} 1$ (between the fault position and $\mathrm{J} 1$ ). 


$$
\begin{gathered}
I_{S}=\frac{U_{a}+U_{A 1 S}}{Z_{A 1 S}+R_{f}+R_{g 1}} \\
I_{R}=\frac{U_{a}+U_{A 1 R}+U_{B 2}+U_{C 3}}{Z_{A 1 R}+Z_{B 2}+Z_{C 3}+R_{f}+R_{g 2}}
\end{gathered}
$$

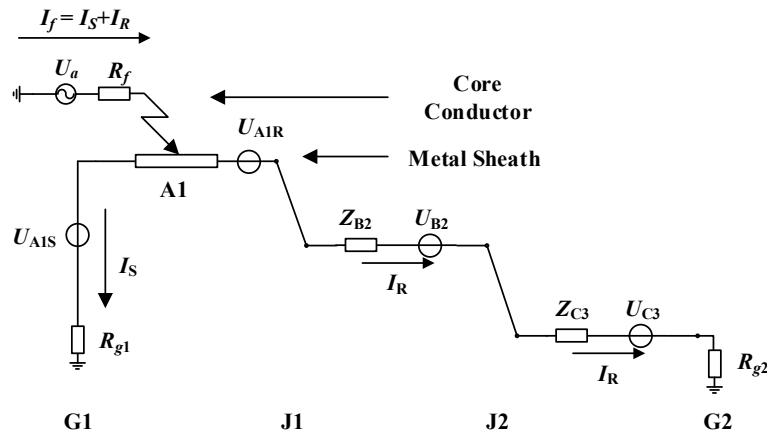

Fig. 4. The schematic diagram of the sheath currents when a short circuit fault occurs in section A1

The analysis which follows will present the differences in the impedance of the metal sheath between fault conditions and non-fault conditions. The fault condition here implies that there is short-circuit fault in the cable section, while the nonfault condition indicates a cable section which does not contain a short-circuit fault, even if there is a through-fault current flowing in the cable conductors. The cause of the change in impedance comes from the change in induced emf in each of the cable sections. For convenience of calculation and expression, (4) and (5) can be represented as (6) and (7), and the sheath current $I_{m 2}$ of the loop B1-C2-A3, the sheath current $I_{m 3}$ of the loop C1-A2-B3 can be represented by (8) and (9), where $E\left(I_{S}\right)$ is the total induced emf in the fault loop $I_{S} ; E\left(I_{R}\right)$ is the total induced emf in the fault loop $I_{R} ; E\left(I_{m 2}\right)$ is the total induced emf in sheath loop B1-C2-A3; $E\left(I_{m 3}\right)$ is the total induced emf in sheath loop $\mathrm{C} 1-\mathrm{A} 2-\mathrm{B} 3 ; R_{s 0}$ is the resistance of the metal sheath per unit length; $R_{c 0}$ is the resistance of the conductor per unit length; $L_{1}$ is the length of sheath loop A1-B2-C3, $L_{2}$ is the length of sheath loop B1-C2$\mathrm{A} 3$, and $L_{3}$ is the length of sheath loop C1-A2-B3.

$$
\begin{gathered}
I_{S}=-\frac{U_{a}+E\left(I_{S}\right)}{R_{g 1}+R_{f}+\left(R_{c 0}+R_{S 0}\right) \times L_{x}} \\
I_{R}=\frac{U_{a}+E\left(I_{R}\right)}{R_{g 2}+R_{f}+R_{c 0} \cdot L_{x}+R_{s 0} \cdot\left(L_{1}-L_{x}\right)} \\
I_{m 2}=\frac{E\left(I_{m 2}\right)}{R_{g 1}+R_{g 2}+R_{s 0} \cdot L_{2}} \\
I_{m 3}=\frac{E\left(I_{m 3}\right)}{R_{g 1}+R_{g 2}+R_{s 0} \cdot L_{3}}
\end{gathered}
$$

For a given cable circuit, the basic circuit and soil parameters can be taken as constants, then $I_{m 2}, I_{m 3}, I_{S}, I_{R}$ become the functions of only 4 variables after all the expressions of the induced emfs are substituted into equations (6) (9). Thus, $I_{m 2}, I_{m 3}, I_{S}, I_{R}$ can be calculated simultaneously. It is to be noted that $I_{S}$ in the fault circuit loop (Fig. 4) and $I_{m 1}$ in sheath circuit loop A1-B2-C3 (Fig. 3) flow in opposite directions: therefore, $I_{S}$ has a negative sign in (6).

\section{THE IMPEDANCE OF THE METAL SHEATH UNDER FAULT AND NON-FAULT CONDITIONS}

\section{A. The Impedance of the Metal Sheath under Non-Fault Condition}

The longitudinal section of a typical HV cable is shown in Fig.5, where $I_{m}$ represents the sheath current, and $I_{c}$ represents the core current. The magnetic induction of the metal sheath comes from both $I_{m}$ and $I_{c}$, represented as $B_{i 2}\left(I_{m}\right)$ and $B_{i 2}\left(I_{c}\right)$. The magnetic induction can be calculated by Ampere's Circuital Law, i.e., the integral of the magnetic induction $B$ around a closed path $C$ equals the magnetic permeability $\mu$ times the current crossing the area bounded by $C$. When a circle of radius $r\left(r_{2}<r<r_{3}\right)$ is chosen to be the closed path, the current enclosing the circle area is $\left(r^{2}-r_{2}^{2}\right) /\left(r_{3}{ }^{2}-r_{2}{ }^{2}\right) \cdot I_{m}$. The equation for determining $B_{i 2}\left(I_{m}\right)$ is represented as (10).

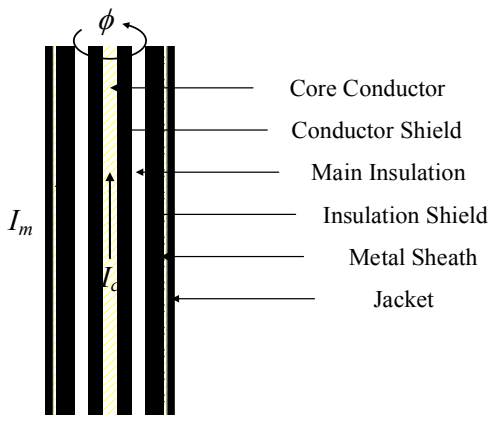

Fig. 5. The longitudinal section of a typical HV cable

$$
B_{i 2}\left(I_{m}\right)=\frac{\mu_{0}\left(r^{2}-r_{2}^{2}\right) I_{m}}{2 \pi\left(r_{3}^{2}-r_{2}^{2}\right) r}
$$

The magnetic flux of the metal sheath $\Phi_{i 2}\left(I_{m}\right)$ is the integral of $B_{i 2}$, which can be expressed as (11).

$$
\Phi_{i 2}\left(I_{m}\right)=\int_{r_{2}}^{r_{3}} \frac{\mu_{0}\left(r^{2}-r_{2}^{2}\right)}{2 \pi\left(r_{3}^{2}-r_{2}^{2}\right) r} I_{m} d r=\frac{\mu_{0} I_{m}}{2 \pi}\left(\frac{1}{2}-\frac{r_{2}^{2}}{r_{3}^{2}-r_{2}^{2}} \ln \frac{r_{3}}{r_{2}}\right)
$$

It is to be noted that the flux linkage $\Psi_{i 2}\left(I_{m}\right)$ linking with the current inside the closed path can be represented as (12).

$$
\begin{aligned}
\Psi_{i 2}\left(I_{m}\right) & =\int_{r_{2}}^{r_{3}} \frac{\mu_{0}\left(r^{2}-r_{2}^{2}\right)^{2}}{2 \pi\left(r_{3}^{2}-r_{2}^{2}\right)^{2} r} I_{m} d r \\
& =\mu_{0} I_{m}\left(\frac{r_{3}^{2}-3 r_{2}^{2}}{8 \pi\left(r_{3}^{2}-r_{2}^{2}\right)}+\frac{r_{2}^{4}}{2 \pi\left(r_{3}^{2}-r_{2}^{2}\right)^{2}} \cdot \ln \frac{r_{3}}{r_{2}}\right)
\end{aligned}
$$

According to Faraday's law of electromagnetic induction, the induced electromotive force of the core conductor per unit length is given as (13).

$$
e_{i 2}\left(I_{m}\right)=-\frac{d \Psi_{i 2}}{d t}
$$

Assuming $i_{m}(t)=I_{\mathrm{AM}} \cdot \sin (\omega t+\theta)$, where, $I_{m}$ is the phasor form of $i_{m}(t) ; I_{\mathrm{AM}}$ is the amplitude of $i_{m}(t) ; \omega$ is the angular frequency of $i_{m}(t), \omega=2 \pi f ; f$ is the frequency of $i_{m}(t) ; t$ is time variable; $\theta$ is time constant. Then the phasor form of $e_{i 2}\left(I_{m}\right)$ can be written as (14) and the self-impedance of the metal sheath is given as (15). 


$$
\begin{gathered}
E_{i 2}\left(I_{m}\right)=-j \mu_{0} f\left(\frac{r_{3}^{2}-3 r_{2}^{2}}{4\left(r_{3}^{2}-r_{2}^{2}\right)}+\frac{r_{2}^{4}}{\left(r_{3}^{2}-r_{2}^{2}\right)^{2}} \cdot \ln \frac{r_{3}}{r_{2}}\right) \cdot I_{m} \\
X_{i 2}\left(I_{m}\right)=\mu_{0} f\left(\frac{r_{3}^{2}-3 r_{2}^{2}}{4\left(r_{3}^{2}-r_{2}^{2}\right)}+\frac{r_{2}^{4}}{\left(r_{3}^{2}-r_{2}^{2}\right)^{2}} \cdot \ln \frac{r_{3}}{r_{2}}\right)
\end{gathered}
$$

Similarly, the induced emf $E_{i 2}\left(I_{c}\right)$ and the mutual impedance $X_{i 2}\left(I_{c}\right)$ can be represented by (16) and (17) respectively.

$$
\begin{gathered}
E_{i 2}\left(I_{c}\right)=-j \mu_{0} f \ln \frac{r_{3}}{r_{2}} \cdot I_{c} \\
X_{i 2}\left(I_{c}\right)=\mu_{0} f \ln \frac{r_{3}}{r_{2}}
\end{gathered}
$$

\section{B. The Impedance of the Metal Sheath under Fault Condition}

The longitudinal section of a typical HV cable under fault condition is shown in Fig.6. The fault current $I_{f}$ (core current) is divided into two parts at the fault position, one part $\left(I_{S}\right)$ flows from the fault position to $\mathrm{G} 1$, and the other part $\left(I_{R}\right)$ flows from the fault position to G2. $I_{S}$ and $I_{R}$ are sheath currents flowing in opposite directions.

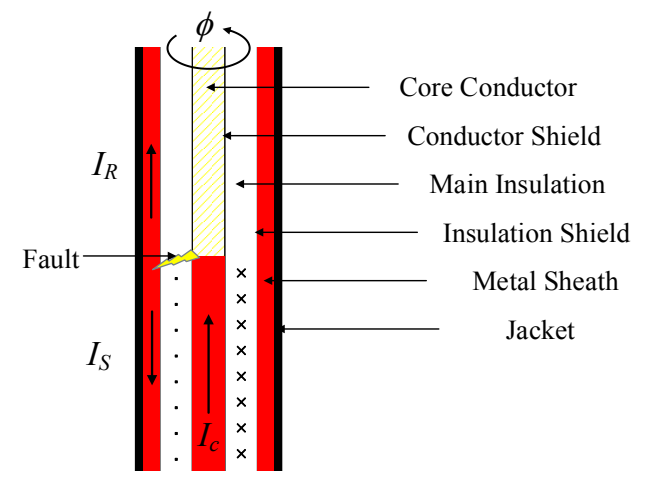

Fig. 6. The current flow in the longitudinal section of a typical HV cable under fault condition

As the complete path of the fault current $I_{S}$ contains both the core conductor and the metal sheath, the flux linkage generated by $I_{S}$ links in the area of the core conductor and the metal sheath, or the self-impedances of circuit $I_{S}$ includes the core conductor self-impedance and the metal sheath selfimpedance. In addition, there are mutual impedances between $I_{S}$ and $I_{R}$, the flux linkage generated by $I_{S}$ and $I_{R}$ links in the main insulation area.

Derived from Ampere's Circuital Law, the induced emf of circuit $I_{S}$, and the self-impedance $X_{i 2}\left(I_{S}\right)$ can be represented by (18) and (19) respectively.

$$
\begin{gathered}
E_{i 2}\left(I_{S}\right)=-j \mu_{0} f\left(\frac{r_{3}^{4}}{\left(r_{3}^{2}-r_{2}^{2}\right)^{2}} \ln \frac{r_{3}}{r_{2}}-\frac{3 r_{3}^{2}-r_{2}^{2}}{4\left(r_{3}^{2}-r_{2}^{2}\right)}\right) \cdot I_{S} \\
X_{i 2}\left(I_{S}\right)=\mu_{0} f\left(\frac{r_{3}^{4}}{\left(r_{3}^{2}-r_{2}^{2}\right)^{2}} \ln \frac{r_{3}}{r_{2}}-\frac{3 r_{3}^{2}-r_{2}^{2}}{4\left(r_{3}^{2}-r_{2}^{2}\right)}\right)
\end{gathered}
$$

Likewise, the mutual impedance between $I_{S}$ and $I_{R}$ can be calculated by (20) and (21).

$$
\begin{gathered}
E_{e 1}\left(I_{R}\right)=-j \mu_{0} f \ln \frac{r_{2}}{r_{1}} \cdot I_{R} \\
X_{i 2}\left(I_{R}\right)=\mu_{0} f \ln \frac{r_{2}}{r_{1}}
\end{gathered}
$$

Under normal, or non-fault conditions, the induced emf of the metal sheath per unit length is shown in (14), and the per unit length self-impedance is shown in (15). Under fault condition, the induced emf of the metal sheath per unit length is given in (20), and the self-impedance of the metal sheath per unit length is shown in (21). Comparing (21) with (15), it can be seen that the per unit length self-impedance of the metal sheath is related to the structure and material of the cable. During a fault, there are changes in the equation of the self-impedance, and in the induced emf.

Changes to the circuit parameters will become even more significant when considering the mutual impedance among the three phase cables. Fig. 7 presents the equivalent circuit when a short-circuit fault occurs in section $\mathrm{B} 2$, where $U_{b}$ is the equivalent voltage source of phase $\mathrm{B} ; U_{B 2 S}$ is the equivalent induced emf on sheath section B2 (between the fault position and $\mathrm{J} 1) ; U_{B 2 R}$ is the equivalent induced emf on sheath section B2 (between the fault position and J2). The fault current $I_{f}$ starts from the power source $U_{b}$, flows along the core conductor of phase B cable (section B1 and part of section B2) to the fault position, it divides into two parts and flows along the metal sheath to the two directly-grounded points. Current $I_{S}$ flows along the core conductor of $\mathrm{B} 1$ and the metal sheath of A1. The positional relationship between the currents $I_{S}$ and $I_{R}$ is shown in Fig. 8, where $I_{m 2}$ is the sheath current of phase $\mathrm{B}$ cable, $d$ is the distance between the neighbouring cables.

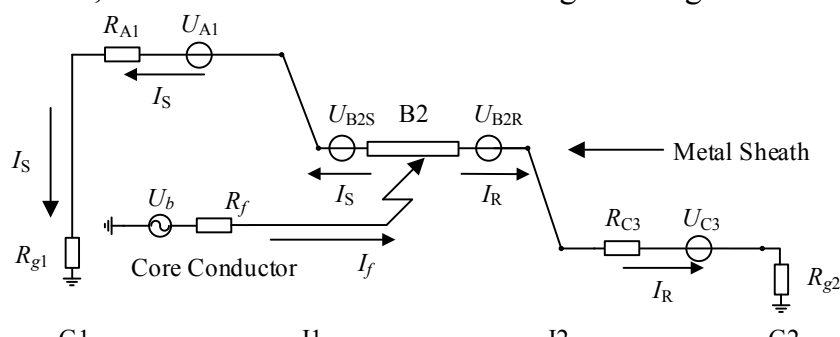

G

$\mathrm{J} 1$

$\mathrm{J} 2$

G2

Fig. 7. The schematic diagram of the sheath currents calculation when a short-circuit fault occurs in section B2

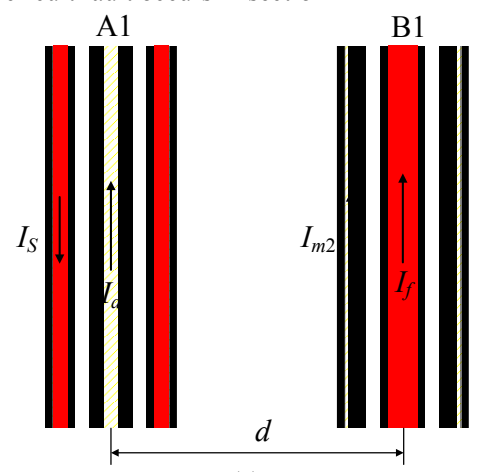

(a)

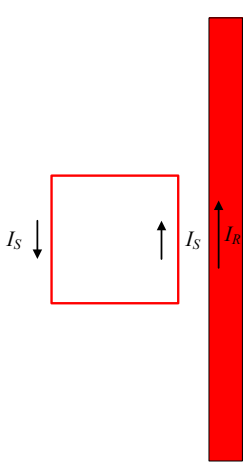

(b)
Fig. 8 The mutual impedance between phases under fault condition $\&$ the equivalent circuit

According to the positional relationship between the currents $I_{S}$ and $I_{R}$, the electromagnetic field connection between $I_{S}$ and $I_{R}$ can be represented as the connection 
between an infinitely long straight current-carrying line $\left(I_{R}\right)$ and a current-carrying coil $\left(I_{S}\right)$, as shown in Fig. 8. The magnetic field generated by $I_{R}$ links with the magnetic field generated by $I_{S}$ in the area of the current-carrying coil, i.e., the area between cable section A1 and cable section B1. The magnetic induction of this area can be represented as $B_{1 e}\left(I_{R}\right)$. Similarly, the induced emf $E_{1 e}\left(I_{R}\right)$ and the self-impedance $X_{1 e}\left(I_{R}\right)$ can be represented as in $(22) \sim(23)$ respectively.

$$
\begin{gathered}
E_{1 e}\left(I_{R}\right)=-j \mu_{0} f \ln \frac{d-r_{3}}{r_{1}} \cdot I_{R} \\
X_{1 e}\left(I_{R}\right)=\mu_{0} f \ln \frac{d-r_{3}}{r_{1}}
\end{gathered}
$$

In conclusion, the equivalent circuits and the cable impedances per unit length are different between the non-fault and fault conditions. The values depend on the position of the fault section, the cable configuration, the cable materials and the relative position between cables of different phases. A revised calculation model is needed to study the cable sheath currents under short-circuit fault conditions.

\section{THE INDUCED EMF IN A CROSS-BONDED HV CABLE SYSTEM UNDER FAULT CONDITION}

This section presents the calculation of the emfs induced by fault current $I_{S}$ in situations which were described in sections $\mathrm{A} \sim \mathrm{C}$. The calculations of the sheath currents in other loops can be carried out in exactly the same way.

\section{A. The Electromotive Forces Induced from Fault Current $I_{S}$}

Assuming a short-current fault occurs in cable section A1, flat laid three phase cables in minor section 1 can be represented as in Fig. 9. $I_{b}$ represents the core current of phase $\mathrm{B} ; I_{c}$ represents the core current of phase $\mathrm{C} ; I_{m 2}$ represents the sheath current of the B1-C2-A3 loop; $I_{m 3}$ represents the sheath current of the $\mathrm{C} 1-\mathrm{A} 2-\mathrm{B} 3$ loop; $I_{S}$ represents the fault current flowing along the core conductor of phase $\mathrm{A}$ to the fault position, and then flowing back through the sheath to the grounding position at G1.

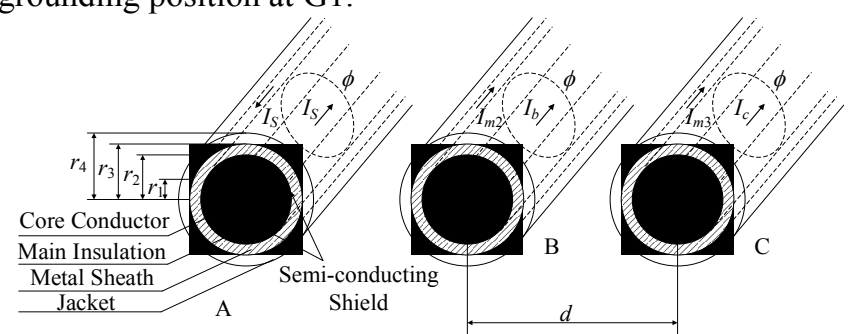

(a) The schematic diagram of fault current flows in minor section 1 (Between G1 and the Fault Position)

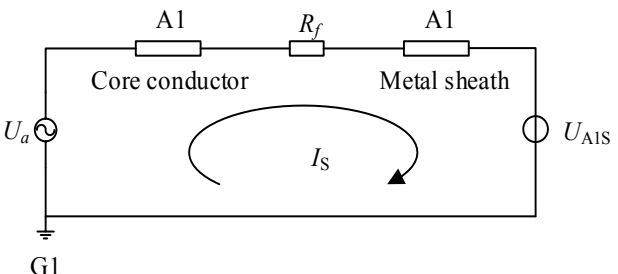

(b) The equivalent circuit diagram of the fault loop in minor section A1 (Between G1 and the Fault Position)

Fig. 9. The schematic diagram of fault currents flowing in minor section $1 \&$ its equivalent circuit diagram
As Fig. 4 and Fig. 9 show, the short circuit fault affects the core and the sheath directly, combining the original core loop with the sheath loop. However, the induced emf can still be calculated by Ampere's Circuit Law and Faraday's law of induction. When the parameters related to the cable structure are available, the changes in magnetic flux can be calculated layer by layer. To calculate the magnetic flux, a closed path is needed to integrate the magnetic induction intensity. This paper chose the concentric circle of the cable cross section as the closed path, of which radius is assumed to be $r$.

1) $0<r<r_{1}$

By assuming the radius of the closed path to be greater than 0 and less than $r_{1}$, the magnetic flux in the core conductor is taken into consideration. The core conductor of a HV cable normally contains multiple stranded wires, which can limit the skin effect. Therefore, the core current can be assumed as surface current. The current inside the closed path is $\left(r^{2} / r_{1}^{2} \cdot I_{S}\right)$. According to Ampere's Circuit Law, the magnetic induction $B_{i 1}\left(I_{S}\right)$ at $r$ is presented as (24).

$$
B_{i 1}\left(I_{S}\right)=\frac{\mu_{0} r}{2 \pi r_{1}^{2}} I_{S}
$$

The magnetic flux $\Phi_{i 1}\left(I_{S}\right)$ of this area can be calculated from (24), and be represented as (25).

$$
\Phi_{i 1}\left(I_{S}\right)=\int_{0}^{\eta_{1}} \frac{\mu_{0} r}{2 \pi r_{1}^{2}} I_{S} d r=\frac{\mu_{0}}{4 \pi} I_{S}
$$

Note that the flux linkage $\Psi_{i 1}\left(I_{S}\right)$ linking with the current inside the closed path can be represented as (26).

$$
\Psi_{i 1}\left(I_{S}\right)=\int_{0}^{r_{1}} \frac{\mu_{0} r^{3}}{2 \pi r_{1}^{4}} I_{S} d r=\frac{\mu_{0}}{8 \pi} I_{S}
$$

According to Faraday's law of induction, the induced emf of the core conductor per unit length is given as (27), the phasor form of $e_{i 1}\left(I_{S}\right)$ can be written as (28).

$$
\begin{gathered}
e_{i 1}\left(I_{S}\right)=-\frac{d \Psi_{i 1}}{d t} \\
E_{i 1}\left(I_{S}\right)=-\frac{j \mu_{0} f}{4} \cdot I_{S}
\end{gathered}
$$

2) $r_{1}<r<r_{2}$

When the radius of the closed path is assumed to be greater than $r_{1}$ and less than $r_{2}$, the magnetic flux in the main insulation is taken into consideration. In this case, the current inside the closed path is $I_{S}$, which fully links with the magnetic flux. Derived from Ampere's Circuital Law, the induced emf of the main insulation per unit length is represented as (29).

$$
E_{e 1}\left(I_{S}\right)=-j \mu_{0} f \ln \frac{r_{2}}{r_{1}} \cdot I_{S}
$$

3) $r_{2}<r<r_{3}$

When the radius of the closed path is assumed to be greater than $r_{2}$ and less than $r_{3}$, the magnetic flux in the metal sheath is taken into consideration. The induced emf of the metal sheath per unit length is provided in (18). Besides the induced emfs from $I_{S}$ itself, there are induced emfs from currents in other phases. 


\section{B. The Electromotive Force Induced from Fault Current $I_{R}$}

The interaction between $I_{S}$ and $I_{R}$ has been analysed in section III.B. The magnetic flux of $I_{R}$ linked with the magnetic flux of $I_{S}$ in the area of the main insulation. The induced electromotive force can be found in (20).

\section{The Induced Electromotive Forces due to Currents of Phase B and Phase C Cables}

When current $I_{b}$ flows in the core conductor of phase B cable, the magnetic flux linking with $I_{S}$ cannot be represented directly by Ampere's Circuit Law as no fully linked loop exists. However, the theory of electric dipoles [24] and Sommerfeld Integral [25] can be used for the calculation. The current element can be treated as an electric dipole, as shown in Fig. 10, and the electric dipole is above the ground with a height of $h$, the distance between the electric dipole and the observation point $P$ is $R_{1}$, the distance between the observation point $P$ and the mirror of the electric dipole is $R_{2}$.

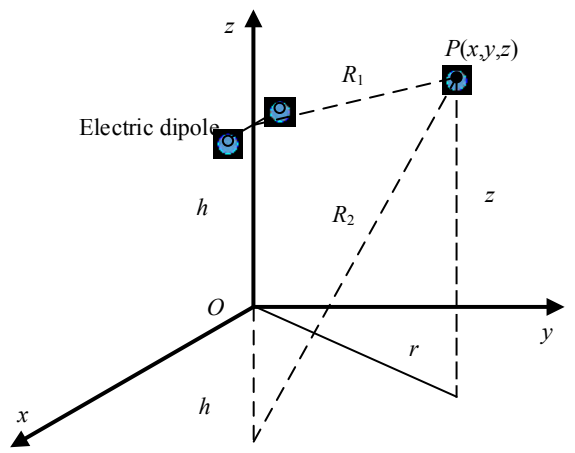

Fig. 10. The configuration of a horizontal electric dipole with the observation point $P$

In an infinite space of non-conducting medium, the component of the Hertz electric vector $\Pi$ generated at the observation point $P(\mathrm{x}, \mathrm{y}, \mathrm{z})$ by the electric dipole in the orientation shown is represented by (30).

$$
\begin{gathered}
\Pi=-\frac{j \omega \mu I d x}{4 \pi k^{2}} \frac{e^{j k R_{1}}}{R_{1}} \\
R_{1}=\sqrt{x^{2}+y^{2}+(h-z)^{2}} \\
\frac{e^{j k R_{1}}}{R_{1}}=\int_{0}^{\infty} \frac{u}{\sqrt{u^{2}-k^{2}}} e^{-\sqrt{u^{2}-k^{2}}|h-z|} J_{0}(r u) d u \\
k=j \sqrt{j \omega \mu(\sigma+j \omega \varepsilon)} \\
r=\sqrt{x^{2}+y^{2}}
\end{gathered}
$$

Equation (31) is the Sommerfeld Integral, where $k, J_{0}(r u)$ and $r$ represents the medium propagation constant, the 0 -order Bessel function of the first kind, the spatial frequency, and the horizontal distance between the electric dipole and the observation point respectively. Assuming plane $x O y$ is the interface between the air (above $x O y$ ) and the earth (under $x O y$ ), the Hertz electric vector in the air is as shown in (32), and the Hertz electric vector in the earth is as shown in (33). The horizontal component of the electric field strength observed in the air and the earth are given by (34) and (35), respectively.

$$
\begin{gathered}
\Pi_{a}=-\frac{j \omega \mu I d x}{4 \pi k_{0}{ }^{2}}\left[\frac{e^{j k_{0} R_{1}}}{R_{1}}-\frac{e^{j k_{0} R_{2}}}{R_{2}}+2 \int_{0}^{\infty} \frac{u}{\beta_{0}+\beta_{1}} e^{-\beta_{0}(z+h)} J_{0}(r u) d u\right] \\
\Pi_{e}=-\frac{j \omega \mu I d x}{4 \pi k_{0}^{2}} \int_{0}^{\infty} \frac{u}{\beta_{0}+\beta_{1}} e^{-\beta_{0} h-\beta_{1}|z|} J_{0}(r u) d u \\
R_{2}=\sqrt{x^{2}+y^{2}+(h+z)^{2}} \\
E_{a}=-\frac{j \omega \mu I d x}{4 \pi}\left[\frac{e^{j k_{0} R_{1}}}{R_{1}}-\frac{e^{j k_{0} R_{2}}}{R_{2}}+2 \int_{0}^{\infty} \frac{u}{\beta_{0}+\beta_{1}} e^{-\beta_{0}(z+h)} J_{0}(r u) d u\right] \\
E_{e}=-\frac{j \omega \mu I d x}{4 \pi} \int_{0}^{\infty} \frac{u}{\beta_{0}+\beta_{1}} e^{-\beta_{0} h-\beta_{1}|z|} J_{0}(r u) d u
\end{gathered}
$$

The cables are assumed to be laid in earth when the induced emfs from other phases are calculated. The induced emf $E_{A B}$ due to current $I_{b}$ can be obtained using (36), and the induced emf between phase $A$ and phase $C$ cables $E_{A C}$ can be calculated using the same method, as shown in (37). Hence, the total induced emf in the fault loop $I_{S}$ can be represented as (38), where $L_{x}$ is the distance between $G 1$ and the fault position.

$$
\begin{gathered}
E_{A B}=-\frac{j \omega \mu I_{b} d x}{4 \pi} \int_{0}^{\infty} \frac{u}{\beta_{0}+\beta_{1}} e^{-\beta_{0} h_{A B}-\beta_{1}\left|z_{A B}\right|} J_{0}(r u) d u \\
E_{A C}=-\frac{j \omega \mu I_{C} d x}{4 \pi} \int_{0}^{\infty} \frac{u}{\beta_{0}+\beta_{1}} e^{-\beta_{0} h_{A C}-\beta_{1}\left|z_{A C}\right|} J_{0}(r u) d u \\
E\left(I_{S}\right)=U_{A 1 S} \\
=\left[E_{i 1}\left(I_{S}\right)+E_{e 1}\left(I_{S}\right)+E_{i 2}\left(I_{S}\right)+E_{e 1}\left(I_{R}\right)+E_{A B}+E_{A C}\right] \cdot L_{x}
\end{gathered}
$$

The total induced emf $E\left(I_{R}\right)$ in the fault loop $I_{R}$, the total induced emf $E\left(I_{m 2}\right)$ in sheath loop B1-C2-A3, and the total induced emf $E\left(I_{m 3}\right)$ in sheath loop C1-A2-B3 can be calculated by the very same method.

\section{A CASE Study OF THE Proposed Model}

A simple example power system is shown in Fig. 11, it contains a power source, a transformer, a major section of cross-bonded cables and a three-phase balanced load. The cables are installed in a flat horizontal formation (the distance between the neighbouring cables $d$ is $0.3 \mathrm{~m}$ ), directly buried into soil at a depth of $1 \mathrm{~m}$. Each minor section of the cable circuit is $500 \mathrm{~m}$ long, hence the total length of the major section is $1500 \mathrm{~m}$. The ground resistance in each of the sheath loops is taken as $0.1 \Omega$. The resistivity of the soil is $100 \Omega \cdot \mathrm{m}$. The cross section of the cable is $800 \mathrm{~mm}^{2}$. The structural parameters are shown in Table I.

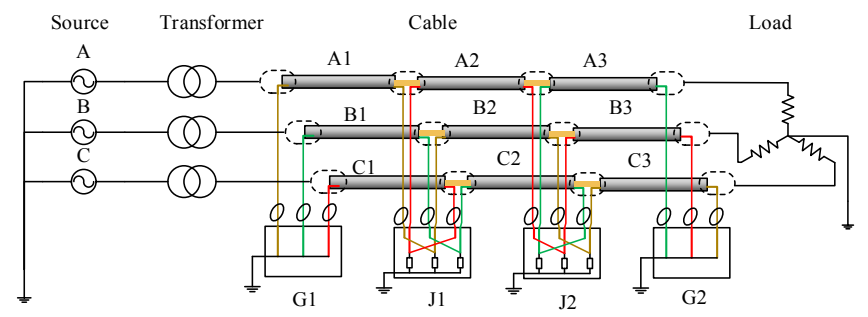

Fig. 11. Schematic diagram of a cross-bonded HV cable system 
TABLE I

Parameters of Cross-sectional Structure of the Cable Structure

\begin{tabular}{ccc}
\hline & Structure & $\begin{array}{r}\text { Outer } \\
\text { radius/mm }\end{array}$ \\
\hline 1 & Core conductor (Copper) & 17.0 \\
2 & Inner semi-conductor (Nylon belt) & 18.4 \\
3 & Main insulation (Ultra-clean XLPE) & 34.4 \\
4 & Outer semi-conductor (Super-smooth semi- & 35.4 \\
& conductive shielding material) & 39.4 \\
5 & Water-blocking layer (Semi-conductor)* & 43.9 \\
6 & Metal sheath (aluminum) & 48.6 \\
7 & Jacket (PVC) & \\
\hline
\end{tabular}

*Note: The water-blocking layer in this cable is made of semi-conductor material, this is equivalent to the outer semi-conductor in the calculation.

The three-phase voltages of the $110 \mathrm{kV}$ power source are taken as $U_{a}=63.51 \angle 0^{\circ} \mathrm{kV}, U_{b}=63.51 \angle-120^{\circ} \mathrm{kV}, U_{c}$ $=63.51 \angle 120^{\circ} \mathrm{kV}$, and the balanced load is $40 \mathrm{MW}$. A singlephase short circuit fault is assumed to occur in cable section A1. According to [26], the breakdown characteristics of XLPE gives an electrical conductivity for the main breakdown channel of around $10^{-3} \Omega \cdot \mathrm{cm}$ and, hence, the resistance of the breakdown channel is around several milliohms. The exact value depends on the cross-section of the breakdown channel. Since the fault resistance is very low in the instant of a breakdown, the fault resistance $R_{f}$ is set as $0 \Omega$ in this study. In this investigation, the fault position $L_{x}$ is set as an independent variable. As the length of section A1 is $500 \mathrm{~m}$, the variation range of $L_{x}$ is $(0,500)$, which means the fault position can be anywhere in section A1. As a result, the fault currents $I_{S}$ and $I_{R}$ can be calculated for any given value of the independent variable $L_{x}$ using the calculation model presented in Section V. The results are presented in Fig. 12 and Fig. 13.

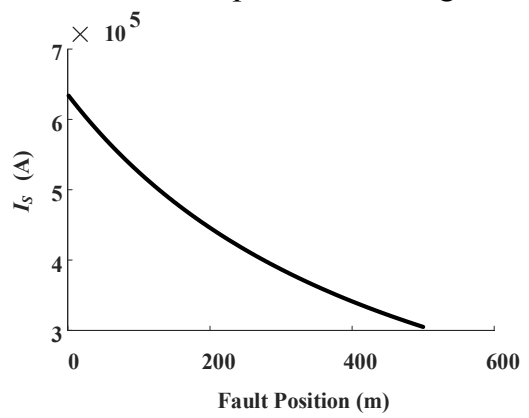

Fig. 12. The relationship between fault current $I_{S}$ and the fault position

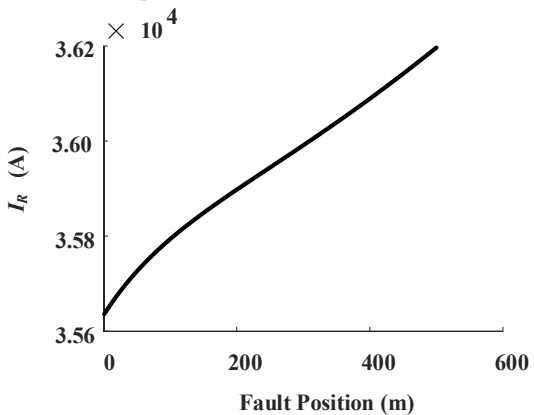

Fig. 13. The relationship between fault current $I_{R}$ and the fault position

Fault currents $I_{S}$ and $I_{R}$ change monotonically with fault position, as shown in Fig. 12 and Fig. 13. It should, therefore, be possible to locate a fault by analysing fault currents $I_{S}$ and $I_{R}$, which can be obtained by monitoring cable sheath currents.

\section{A Comparison of SEVERAL Popular Methods FOR THE CALCULATION OF PHASE DifFERENCE AND FAULT LOCATION}

In order to compare the differences among calculation methods, simulations have been carried out where a fault was set in cable section $\mathrm{A} 1, \mathrm{~B} 2$ or $\mathrm{C} 3$, in turn. In addition to the proposed method, three other methods have been included in the simulation calculations. The methods are: the Pollaczek's method [27] used in PSCAD [28], the calculation method recommended in a CIGRE technical brochure [29], and the calculation method without considering the mutual impedance under fault conditions. The simulation results are provided in Fig. 14.

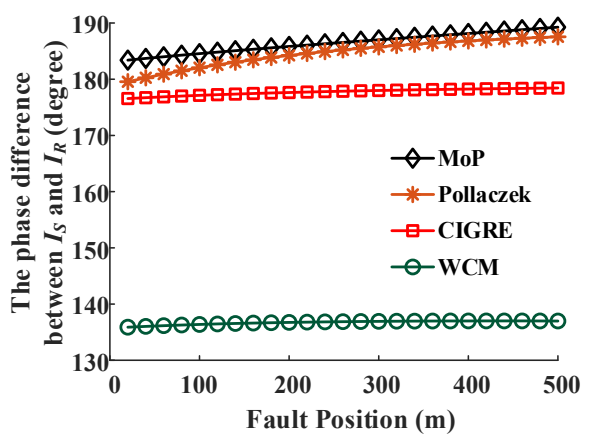

(a) The phase difference between fault current $I_{S}$ and fault current $I_{R}$ when the fault occurs in cable section A1

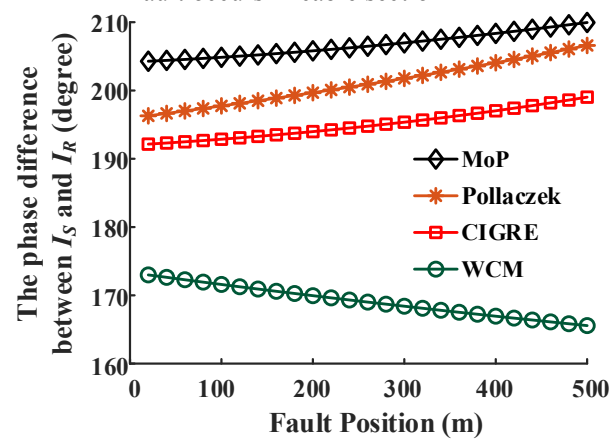

(b) The phase difference between fault current $I_{S}$ and fault current $I_{R}$ when the

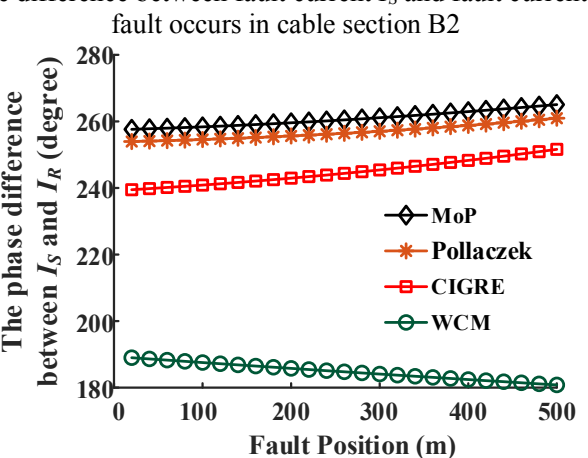

(c) The phase difference between fault current $I_{S}$ and fault current $I_{R}$ when the fault occurs in cable section $\mathrm{C} 3$

Fig. 14. The result comparison of the 4 calculation methods (MoP is the method of this paper; Pollaczek is Pollaczek's method; CIGRE is the method recommended in a CIGRE technical brochure; WCM is the method without considering the mutual impedance under fault conditions)

As shown in Fig. 14, the phase difference between the fault current $I_{S}$ and fault current $I_{R}$ obtained using the three methods are all different. The results of the Pollaczek's 
method are the closest to the results of the proposed method in this paper. The results of the CIGRE method are the second closest. The results for the method which does not consider the mutual impedance under fault conditions differs significantly from the other methods. This indicates that the mutual impedance under fault conditions plays an important role in the model and should not be ignored. Furthermore, the degree of difference in the results is related to the minor section in which the fault is located. If the phase difference between $I_{S}$ and $I_{R}$ is taken as the criterion for fault location, the errors for the fault location of the 4 methods are shown in Fig. 15.

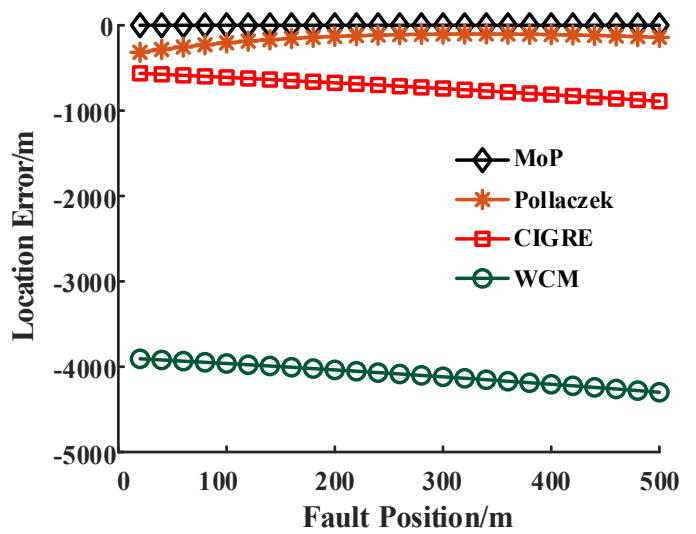

(a) The location errors of the 4 methods when the fault is in cable section A1

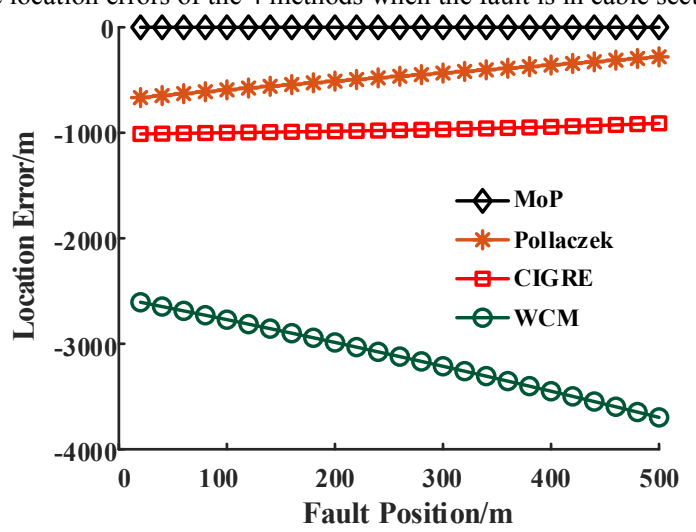

(b) The location errors of the 4 methods when the fault is in cable section B2

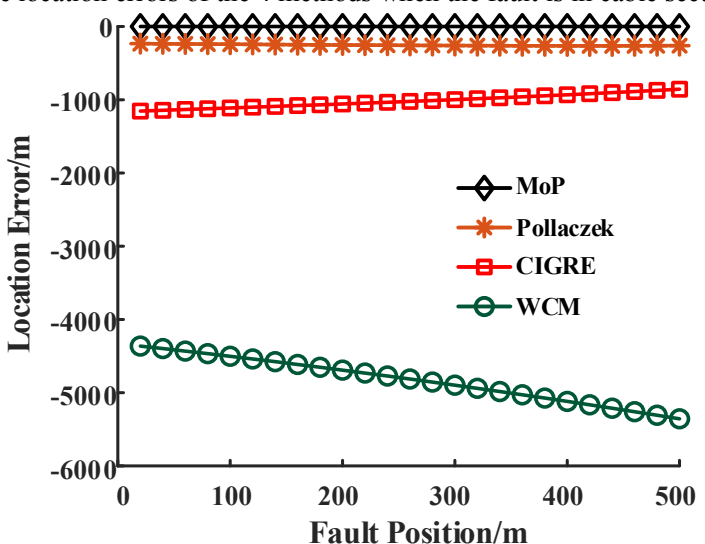

(c) The location errors of the 4 methods when the fault is in cable section $\mathrm{C} 3$ Fig. 15. The comparison of the location errors for the 4 calculation methods

Fig. 14 and Fig. 15 indicate that the phase difference is very sensitive to the fault distance. A small error in the phase difference could cause a large error in estimation of the fault location. The maximum absolute error of each method in each cable section is presented in Table II.

TABLE II

The maximum absolute error* of each method in each cable section

\begin{tabular}{rlll}
\hline Section & Method & CIGRE & WCM \\
\hline A1 & 316.4 & 889.7 & 4299.0 \\
A2 & 680.3 & 1110.0 & 1179.4 \\
A3 & 269.9 & 3852.6 & 5398.9 \\
B1 & 340.7 & 899.2 & 4503.2 \\
B2 & 696.2 & 1211.0 & 1220.3 \\
B3 & 285.4 & 4097.0 & 5550.7 \\
C1 & 314.8 & 885.7 & 4246.1 \\
C2 & 676.5 & 1022.6 & 1154.0 \\
C3 & 263.6 & 3965.1 & 5356.0 \\
\hline
\end{tabular}

*Note: The unit of the error is $\mathrm{m}$.

As shown in Table II, the location error varies with the minor section in which the fault is located. As discussed in section IV, the calculation of the mutual impedance under fault conditions is dependent on the minor section in which the fault is located. Except for the method proposed by the paper, in which the error is $0 \mathrm{~m}$, the fault location errors of the other 3 methods are too large to be accepted in practical situations.

As noted earlier, one of the assumptions made in section $\mathrm{V}$ is that the fault resistance $R_{f}$, the resistance of the breakdown channel, is set to $0 \Omega$, as the value for XLPE is said to be very small. However, there are practical cases [30] that indicate that the current sensors may be triggered before the penetrating breakdown event occurs, in which case the fault resistance $R_{f}$ will not be close to $0 \Omega$. There are two possible solutions to this issue: first, and simplest, solution is to change the triggering and sampling rules of the current sensors; the second solution is to add another criterion for the fault location when the fault resistance $R_{f}$ cannot be ignored.

Although the specific value of the fault resistance $R_{f}$ is not available before the breakdown, the value of $R_{f}$ can be estimated by the fault resistance compensation calculation using the instantaneous power. Based on $[8,31]$, the equation is presented in (39), where $P_{a}$ represents the is the power measured at the power sending end, and $k$ is the rate of $I_{R} / I_{S}$. Hence, the fault still can be located by the phase difference, as presented in Fig. 14 and Fig. 16.

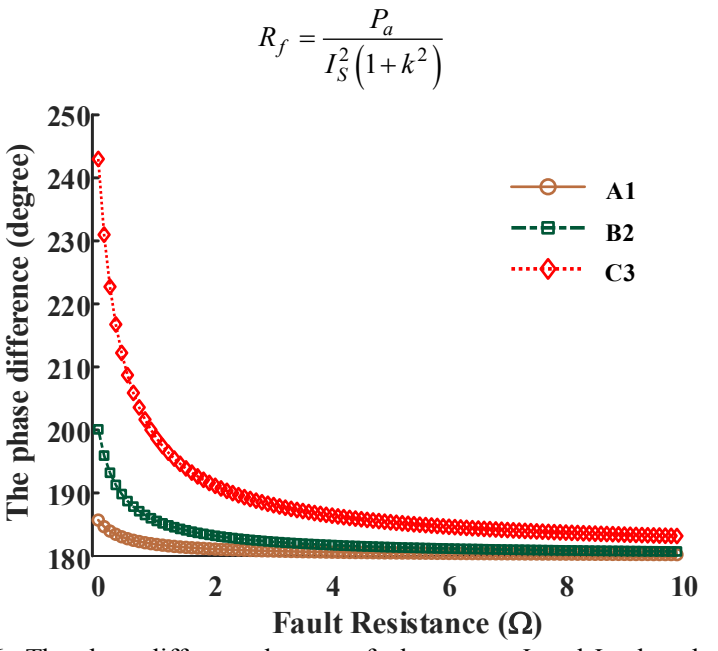

Fig. 16. The phase difference between fault currents $I_{S}$ and $I_{R}$ when the fault resistance cannot be ignored (A1 is the fault occurring in minor section A1; 
$\mathrm{B} 2$ is the fault occurring in minor section B2; C3 is the fault occurring in minor section $\mathrm{C} 3$ )

Fig. 16 indicates that the phase difference between the fault currents $I_{S}$ and $I_{R}$ decreases with the increase of the fault resistance. As a result, the larger the fault resistance becomes, the more difficult it is to distinguish the phase difference. The results also indicate that the accuracy of the current sensors should match the value of the fault resistance to ensure ability to distinguish phase difference values effectively.

\section{CONCLUSIONS AND DISCUSSIONS}

This paper proposes a revised numerical model of $\mathrm{HV}$ cable sheath current under short-circuit fault condition that is applicable to HV cross-bonded cable systems. The differences between fault condition and non-fault condition lie in the equivalent circuit and the cable impedance per unit length. Essentially, the differences result from changes due to the presence of a short-circuit fault, i.e. to the electrical connections and coupling between the cable conductors and the cable sheaths. The paper demonstrated that it is important to not ignore the mutual impedance under fault conditions when the accuracy of the simulated fault current matters in an application.

The method proposed in this paper has been compared with the Pollaczek's method used in PSCAD, an approximate calculation method recommended in a CIGRE technical brochure and the calculation method without considering the mutual impedance under fault conditions. Results show that the method developed is very accurate and that fault location errors of the other 3 methods are too large to be acceptable. The results also indicate that the phase difference is very sensitive to the fault distance: a small error in the phase difference could cause a large error in the fault location.

The phase difference between the fault currents $I_{S}$ and $I_{R}$ decreases with increasing fault resistance. A large fault resistance, if not considered correctly, could affect the ability to distinguish the phase difference. To overcome this challenge, it is suggested that the current sensors be set to trigger when a penetrating breakdown forms, or the accuracy of the current sensors should be matched to the fault resistance. The details of the location method, utilizing the modelling results, will be presented in part 2 of the paper.

\section{REFERENCES}

[1] B. Sheng, W. Zhou, J. Yu, S. Meng, C. Zhou, D. M. Hepburn, "On-line PD detection and localization in cross-bonded HV cable system," IEEE Trans. Dielectr. Electr. Insul., vol. 21, no. 5, pp. 2217-2224, Oct. 2014.

[2] X. Zhang, "Estimation of the Lifetime of the Electrical Components in Distribution Networks," IEEE Trans. Power Del., vol. 22, no.1, pp. 515522, Jan. 2007.

[3] Z. Tang, C. Zhou, W. Jiang, W. Zhou, X. Jing, J. Yu, et al. "Analysis of Significant Factors on Cable Failure Using the Cox Proportional Hazard Model,” IEEE Trans. Power Del., vol. 29, no. 2, pp. 951-957, Apr. 2014.

[4] X. Dong, Y. Yuan, Z. Gao, C. Zhou, B. Sheng, H. Zhao, "Analysis of cable failure modes and cable joint failure detection via sheath circulating current", in EIC, Philadelphia, 2014, pp. 294-298.

[5] A. R. Almeida, O. M. Almeida, B. F. S. Junior, L.H.S.C. Barreto, A. K. Barros, "ICA feature extraction for the location and classification of faults in high-voltage transmission lines," Electric Power Systems Research, vol. 148, pp. 254-263, Jul. 2017.
[6] C. Zhou, Y. Yang, M. Li, W. Zhou, "An integrated cable condition diagnosis and fault localization system via sheath current monitoring," in 2016 International Conference on Condition Monitoring and Diagnosis, Xi'an, China, 2016, pp. 1-8.

[7] Hanif Livani, Cansin Yaman Everenosoglu, "A single-ended fault location method for segmented HVDC transmission line," Electric Power Systems Research, vol. 107, pp. 190-198, Feb. 2014.

[8] M. Eissa, "Ground distance relay compensation based on fault resistance calculation" IEEE Trans. Power Del., vol. 29, no. 4, pp. 1830-1835, Oct. 2006.

[9] Z.Xu, S.Jiang, Q.Yang, T.Bi, “Ground distance relaying algorithm for high resistance fault," IET Gener. Transm. Distrib., vol. 4, no.1, pp. 2735, Jan. 2010.

[10] J.Suonan, J. Qi, "An accurate fault location algorithm for transmission lines based on R-L model parameter identification," Electric Power Systems Research, vol. 76, no. 1-3, pp. 17-24, Sep. 2005.

[11] D. Novosel, D.G. Hart, E. Udren, J. Garitty, "Unsynchronized twoterminal fault location estimation," IEEE Trans. Power Del., vol. 11, no. 1, pp. 130-138, Jan. 1996.

[12] A.A. Girgis, C.M. Fallon, "Fault location technique for radial and loop transmission systems using digital fault recorded data," IEEE Trans. Power Del., vol. 7, no. 4, pp. 1936-1945, Oct. 1992.

[13] T. Ji ,T. Sun, B. Xu, P. Chen, Y. Xue, "Study on fault location of distribution mixed feeders based on double terminal method of traveling waves," Proceedings of the CSEE, vol. 26, no. 12, pp. 89-94, Jun. 2006.

[14] M. Bawart, M. Marzinotto, G. Mazzanti, "Diagnosis and location of faults in submarine power cables", IEEE Elect. Insul. Mag., Vol. 32, No. 4, pp. 24-37, Jul./Aug. 2016

[15] H. M.Manesh, G. Lugrin, R. Razzaghi, C. Romero, M. Paolone, F. Rachidi, "A new method to locate faults in power networks based on electromagnetic time reversal," in IEEE SPAWC, Cesme, Turkey, 2012, pp. 469-474.

[16] Reza Razzaghi, Gaspard Lugrin, Hossein Manesh, Carlos Romero, Mario Paolone, Farhad Rachidi, "An efficient method based on the electromagnetic time reversal to locate faults in power networks," IEEE Trans. Power Del., vol. 28, no. 3, pp. 1663-1673, Jul. 2013.

[17] G. Lugrin, R. Razzaghi, F. Rachidi and M. Paolone, "Electromagnetic time reversal applied to fault detection: The issue of losses," 2015 IEEE International Symposium on EMC, Dresden, 2015, pp. 209-212.

[18] R. Razzaghi, G. Lugrin, F. Rachidi, M. Paolone, "Assessment of the Influence of Losses on the Performance of the Electromagnetic Time Reversal Fault Location Method," IEEE Trans. Power Del. , vol. 32, no. 5, pp. 2303-2312, Oct. 2017.

[19] M. Marzinotto, G. Mazzanti, "The Feasibility of Cable Sheath Fault Detection by Monitoring Sheath-to-Ground Currents at the Ends of Cross-Bonding Sections," IEEE Trans. Ind. Appl., vol. 51, no. 6, pp. 5376-5384, Nov. 2015.

[20] Schelkunoff, S. A. "The Electromagnetic Theory of Coaxial Transmission Lines and Cylindrical Shields," Bell Labs Technical Journal. vol. 13, no. 4, pp. 532-579, Oct. 1934.

[21] L. M. Wedepohl, D. J. Wilcox, "Transient analysis of underground power-transmission systems. System-model and wave-propagation characteristics," Proceedings of the Institution of Electrical Engineers, vol. 120, no. 2, pp. 253-260, Feb. 1973.

[22] L. M. Wedepohl, H.V. Nguyen, G.D. Irwin, "Frequency-Dependent Transformation Matrices for Untransposed Transmission Lines using Newton-Raphson Method," IEEE Trans. Power Syst., vol. 11, no. 3, pp. 1538-1546, Aug. 1996.

[23] IEEE Guide for Bonding Shields and Sheaths of Single-Conductor Power Cables Rated $5 \mathrm{kV}$ through $500 \mathrm{kV}$, IEEE Standard, 2014.

[24] R. Vaughan, J. Bach Anderson, "Channels, propagation and antennas for mobile communications". IET Electromagnetic Waves Series, London, 0 $852960840,2006$.

[25] Sommerfeld A., "Partial differential equations in physics," New York: Academic Press Inc., 1949.

[26] A.S. Vaughan, I.L. Hosier, S.J. Dodd, S.J. Sutton, "On the structure and chemistry of electrical trees in polyethylene", J. Phys. D: Appl. Phys., vol. 39, no. 3, pp.962-978, Jun. 2006.

[27] F. Pollaczek, "Sur le champ produit par un conducteur simple infiniment long parcouru par un courant alternatif", Revue Gén, Elec., vol. 29, pp. 851-867, 1931.

[28] EMTDC User's Guide v4.6, Available: https://hvdc.ca/knowledgebase/read,article/163/emtdc-user-s-guide-v4-6/v:. 
[29] CIGRE: 'CIGRE Technical Brochure 283 on Special Bonding of High Voltage Power Cables', 2005.

[30] Y. Liao, Y. Li , K. Qi, C. Xiang, "Pre-Location Approach for $10 \mathrm{kV}$ High Resistance Cable Fault Based on Improved Bridge Method," Advances of Power System \& Hydroelectric Engineering, vol. 24, no. 5, pp. 47-50, May 2008.

[31] Leif Eriksson, Murari Mohan Saha, G. D. Rockefeller, "An Accurate Fault Locator with Compensation for Apparent Reactance in the Fault Resistance Resulting from Remote-End Infeed," IEEE Power Eng. Rev., vol. PAS-104, no. 2, pp. 424-435, Feb. 1985.

\section{BIOGRAPHIES}

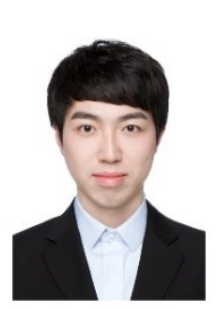

Mingzhen Li (S'17) was born in Xiangyang, China in 1991. He received the B.Sc. degree in Electrical Engineering from Wuhan University, Wuhan, China, in 2014. He is now pursuing the Ph.D degree in Electrical Engineering from Wuhan University, Wuhan, China.

His research is mainly focused on fault location and condition monitoring of power cables.

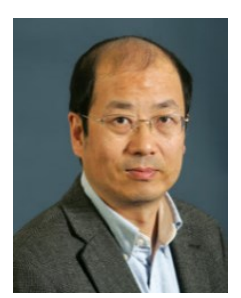

Chengke Zhou (M'06, SM'13) received the B.Sc. and M.Sc. degrees in Electrical Engineering from Hua Zhong University of Science and Technology, China in 1983 and 1986, respectively, and the Ph.D. degree at the University of Manchester U.K., in 1994. Since then, he worked in Glasgow Caledonian University (GCU), as a Lecturer and Senior Lecturer, and in Heriot-Watt University as a Reader. In 2007 he returned to GCU as a Professor. He has published more than 130 papers in the area of PD based condition monitoring of MV/HV plant and power system analysis. He is senior member of IEEE and Fellow of IET.

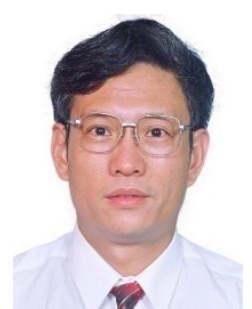

Wenjun Zhou (SM'13) was born in China in July, 1959. He received the Ph.D. degree in hydraulic and electrical engineering from Wuhan University of Hydraulic and Electrical Engineering, Wuhan, China, in 1990. Currently, he is a professor at the School of Electrical Engineering, Wuhan University. He is also the Vice Director of the Hubei High Voltage Committee. His research interests include lightning protection and the diagnostic techniques for outdoor electrical insulations. Dr. Zhou is a member of the High Voltage Committee of the Chinese Society of Electrical Engineering (CSEE), the Electro-technical Test and Measurement Committee of the China Electro-technical Society (CES), and the China Lightning Protection Standard Committee. 